\title{
Research on Shared Express Delivery Mode Based on Block Chain Technology
}

\author{
Ding Yuanyuan, $\mathrm{He} \mathrm{Xu}$ \\ Guizhou University of Finance and Economics \\ Guiyang, Guizhou
}

\begin{abstract}
The rise of sharing economy has remolded the industry form, and express delivery has achieved rapid development on the express train of sharing economy, sharing express came into being at the historic moment. By analyzing the existing shared express mode, the paper concludes the common business mode of shared express, and exposes some problems such as information island, imperfect identity authentication, user privacy security can not be guaranteed, and low efficiency of payment and settlement in the shared express mode. Through the research of block chain technology, a new mode of sharing express delivery based on block chain technology is constructed. It is found that the optimized block chain technology + shared express mode based on the technical characteristics of block chain can effectively solve the pain points of some industries and guarantee the efficient operation of the shared express platform.
\end{abstract}

Keywords-Block chain; Shared express; Shared economic

\section{INTRODUCTION}

With the rapid development of e-commerce, the express logistics industry has achieved unprecedented development. The express logistics industry naturally has shared genes, and the operation mode of shared express has emerged. The rise of shared express has injected fresh vitality into the industry. Various sharing modes, such as product sharing of express logistics, information sharing of express management, end resource sharing, passenger transport resource sharing, Renren express and shared express box, have emerged one after another, but new problems have also emerged. Block chain technology has many advantages such as decentralization, distributed accounting, intelligent contract, and traceability which cannot be altered.. The emergence of block chain provides a good idea for solving some pain points in shared express delivery. If the traditional mode can be reformed and optimized, the utilization rate of resources can be effectively improved,and it will reduce costs and increase efficiency and promote the sustainable development of shared express industry.

\section{ANALYSIS ON THE EXISTING MODE OF SHARING EXPRESS DELIVERY FROM THE PERSPECTIVE OF SHARING ECONOMY}

\section{A. Express logistics technology and product sharing}

The technology and products formed by the logistics industry of traditional express delivery can only reduce the waste of resources from the way of leasing. In our country, most of the logistics technology and product resources in express industry are owned by enterprises alone, andcthey are not generally open and shared as shared resources With the development of technology, people's concept of cooperation has changed, and related technology and product resources can be shared to improve the utilization rate. In practice, pallet sharing is taken as an example to form a recycling sharing loop. Through sharing, pallets are reused in the logistics chain, thus improving the utilization rate of pallets and effectively saving resources.

\section{B. Sharing of end distribution resources}

With the help of third-party express collection platform to achieve sharing. The third-party collection platforms that people are familiar with include Cainiao Courier station, Haidan, Landian and so on ${ }^{[1]}$. The enterprise aims at solving end distribution problem in the express industry more efficiently and trying to establish a third-party express service platform that facilitates end distribution delivery in the community.

With the help of self-lift express cabinet to achieve sharing. The common self-lift express cabinet facilities in the market include easy mail cabinet, neighboring treasure, Fengchao, etc., which belong to independent express service enterprises.

Share by means of end joint distribution. The form of joint distribution is the combination of third-party logistics and users to provide the operation mode of end express logistics distribution under the joint action.,thus the scale effect and synergies can achieve operational goals of reducing costs and increasing efficiency, reducing waste and improving resource utilization from joint distribution.

Resource sharing by passenger transport. Taking advantage of the spare capacity of passenger cars, such as the spare of suitcases. At the beginning and end of passenger transport, arranging the staff to pick up and send pieces respectively, and logistics transportation such as parcels is carried out by sharing transportation capacity.

\section{STUDY ON THE BOTTLENECK RESTRICTING THE DEVELOPMENT OF SHARED EXPRESS}

\section{A. Construction of a common business model for shared delivery}

The traditional operation mode of express delivery not only causes a large amount of waste of resources, but also leads to the sluggish and inefficient operation of all links. By analyzing the characteristics of the existing shared express mode, the common model of shared express delivery is constructed.As shown in Figure. 
The shared express platform connects the suppliers and the demanders, information, centralized data, product technology, end-shared distribution resources and social idle resources and they are integrated on the shared express platform, and the platform can reasonably plan and allocate resources uniformly. The shared express delivery platform strengthens the connection with both suppliers and demanders through information transmission, which requires the information chain to be safe, open,traceable and untouchable.. High-frequency trading will occur when both suppliers and demanders flow funds to the shared express platform at the same time., which requires that the capital chain must be settled conveniently and transparently.In this way, both on-line and off-line suppliers and demanders can have benign interaction on this platform to truly achieve sharing. From the practical situation, it is difficult to trace the information island in the information chain, the identity authentication system is not perfect, and the user privacy security is not protected. The payment and settlement problems in the capital chain have an increasingly prominent impact on the construction of the shared express delivery platform. If these problems are not solved, the platform will not run smoothly and the cost efficiency will not be improved.

\section{B. An island of information is hard to trace}

In the whole trace process, logistics operation points need to be reported layer by layer. The process is long, time-consuming, inefficient and consumes a lot of manpower and material resources. The development of e-commerce has driven not only the prosperity of trade but also the popularity of fake goods market. In addition to social and ethical factors, it is difficult to trace products and distinguish between true and false information that gives illegal traders the opportunity to take advantage of. In the chain of huge commercial interests, each of us may become a victim.

\section{Identity becomes a problem}

Express delivery has become a new channel for crimes. In recent years, the use of express delivery for illegal and criminal activities have occurred frequently, such as firearms and other contraband can be delivered by express delivery, so that its disassembled parts can be transported, and then successfully assembled into firearms after arriving at the destination. Express delivery provides a new medium for crime. The network platform + express delivery provides a new transportation channel for drugs, contraband and dangerous goods. Criminals purchase and reuse express delivery through the virtual platform for transportation, and finally assemble a series of criminal activities ${ }^{[2]}$.

\section{User privacy security issues}

Privacy information leakage caused economic losses to users. Bad-minded criminals deceive users by pretending to be public security organs or bankers after mastering their privacy information. Usually in the form of posing as someone else requires the victim to report the loss of a bank account, credit card transfer to the user to bring economic losses. The disclosure of privacy information of express users not only causes property damage but also seriously affects the personal safety of users, and even poses a great threat to national security. China's Internet users suffer an economic loss of 91.5 billion yuan due to the disclosure of privacy information in one year ${ }^{[3]}$. The development of express delivery industry not only provides convenience for people, but also increases the risk of information leakage, which facilitates the criminal activities downstream of the industrial chain.

\section{Block Chain TeChNOLOGY Basic Model AND TECHNICAl ADVANTAgeS}

\section{A. Basic model analysis of block chain}

The basic block chain model is divided into five levels, as shown in figure 3: data layer, block technology layer, network support layer, consensus incentive layer and practical application layer. Each technology layer claims to be a system and has its own specific operating mechanism. These mechanisms interact and form a huge technical advantage ${ }^{[4]}$.In the specific application of block chain, the model at the corresponding level will change randomly, and various coping methods will be arranged and combined among different levels as the actual situation changes.

Data layer: transaction information with a special format is the most important component of block chain data layer.Block layer: since the hash value in the final parent block is retained, it can only be uniquely recognized after several hash operations, and each block forms a chain structure. The function of block head is to mark the position of block in the whole block chain and guarantee the legitimacy of block. Network layer: the network layer is composed of the connection mode, information transmission mechanism and information verification technology of each network node within the system. Consensus incentive layer: The number of nodes in a typical block chain system is large and their status is completely equal.. Application layer: it can be understood as the presentation of specific business logic on block chain system, including various script codes of the system and intelligent contract application blocks based on $\mathrm{it}^{[5]}$.

\section{B. Block chain technology advantages}

Decentralization. The central mechanism has a unique position in the system, and it can master the distribution information of all nodes. Once the central mechanism is attacked, the whole system will be paralyzed, and the trust mechanism within the system will collapse. The biggest technical advantage of block chain is decentralization, It does not need central control and realizes direct point-to-point interaction, which makes information interaction more efficient and secure.. Even if a node is attacked, it will not affect the operation of the whole system, ensuring the safety and efficiency of information transmission.

Do not be tampered with. The block chain system realizes the interlocking of all the data on the chain by relying on the Merkle tree data structure in the block and the way of stamping the time stamp between blocks and the hash value of the previous block. At the same time, it relies on the consensus mechanism of the whole network to ensure the ultra-high computing power or ultra-high rights and interests requirements of data tampering, so as to ensure that all the datas on the chain are very difficult to be tampered with in a malicious way ${ }^{[5]}$. 
Transactions are transparent and anonymous.Block chain operation rules are open and transparent, and data information is also publicly broadcast in the whole network. Every transaction information is recorded by each node. Due to the decentralized nature of the equal status among all nodes, there is no need to disclose identity information. Each participating node can upload transaction information anonymously, which can effectively guarantee the privacy security of users ${ }^{[6]}$.

\section{A New Mode OF SHAREd EXPRESS DELIVERY BASED ON BLOCK CHAIN TECHNOLOGY}

\section{A. Shared express model under block chain technology}

The most important chain structures supporting the operation of the shared express delivery platform are the information chain and capital chain. Information generation and the whole network broadcasting are publicly preserved in a block. The input of each piece of information after a series of hash operations to retain the hash value to ensure that the information can not be tampered with; the consensus mechanism of block chain can effectively guarantee the security of information. In the capital chain, the unique smart contract of block chain can automatically complete the transaction settlement, while the chain structure and block chain network technology layer jointly maintain the openness and transparency of the account. The new operation mode of shared express is shown in figure:

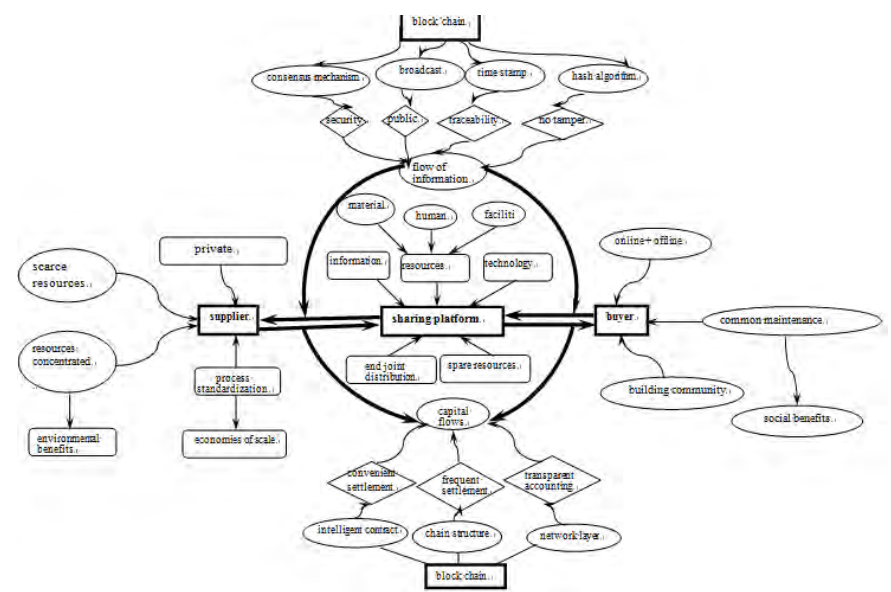

Fig. 1 New mode of Shared express delivery based on block chain technology

\section{B. With the application mode of block chain}

Electronic storage mode. Every express logistics transaction is uploaded to the whole network from the moment it is issued, it can store relevant transaction records in any form, such as pictures, files, videos, etc. into open and distributed storage block chain system. Depositing certificate is a one-time process. After depositing certificate, data transaction can be carried out by means of point-to-point data encryption and interchange. This type of transaction ensures that data can only be decrypted upstream and downstream of the direct docking. The process of concluding digital contracts for a limited number of parties through a process (i.e., sequencing).

Authentication mode. An independent individual, company and organization will separately generate a uniquely identifiable ID for unified authentication on the block chain, and the information related to the identity ID will be stored on the distributed whole network block. On this basis, every behavior related to express delivery will be recorded by the whole network.

Each data block in the payment and settlement supported by block chain technology contains all the network transaction information in the past period of time, which can be used to verify the validity of transaction information and achieve anti-counterfeiting effect..At the same time, the next data block will be generated. E-commerce payment mode constructed by block chain technology can realize all-weather payment and fast account arrival, and reduce the risk of e-commerce funds.

\section{CONCLUSION}

The rise of shared express has realized the economic benefits of centralized allocation of resources, full utilization of idle resources, cost reduction and increasing of.efficiency The new mode of shared express based on block chain technology is helpful to solve the pain points of some industries. Block chain technology can effectively solve outstanding problems such as non-disclosure and non-sharing of information in shared express delivery, difficulty in implementing real-name authentication, leakage of user privacy, complicated payment and settlement by virtue of its many technical advantages such as decentralization, distributed accounting, non-tampering and traceability, intelligent contract etc. Introducing block chain into express delivery industry is a new exploration of the development of express delivery industry, which is bound to have broad prospects in the future.

\section{REFERENCES}

[1] Luo L W. "Platform game". China logistics and procurement, 2015(17):33-35

[2] Wu J T, Le J G. "The present situation of express delivery as the medium of crime and its prevention and control countermeasures are analyzed. Rule of law and society,2018(05).

[3] Gen Y, Sun J F. Express real name system under the user privacy disclosure risk prevention. China's current economy, 2017(11).

[4] Kshetri.N. Block chain's roles in strengthening cybersecurity and protecting privacy. Telecom-municationsPolicy, 2017.

[5] Jiang H F. Research on the application mode of block chain technology in the financial industry. Master's thesis of zhejiang university, 2018.

[6] Li L F. Research on the new pattern of international trade settlement based on block chain technology. Master's thesis of Beijing university of posts and telecommunications, 2018 .

[7] Wang Z H. Analysis on the application of block chain technology in e-commerce network consumption interaction. The electronic commerce, 2017 